\title{
Violencia de Género: nuevas realidades y nuevos retos
}

\section{Gender Violence: new realities and new challenges}

José Manuel Peixoto Caldas

Médico y Sociólogo. Doctor en Sociología y Doctor en Salud Pública. Docente de la Universidad de Barcelona y Consultor del Instituto Cooperación Iberoamericana.

Dirección: Travesera de Dalt, 69, Código Postal 08024, Barcelona, España.

Correo Electrónico: jpeixoto®uoc.edu

\section{Kleber Mauricio Gessolo}

Psicólogo Clínico. Especialista en Psicopedagogía. Psicólogo, Prefectura de Matão - SP.

Dirección: Calle Oreste Bozelli, II65, Centro, CEP 15990-240, Matão, SP, Brasil.

Correo Electrónico: kmgessolo®uol.com.br

\section{Resumen}

Este artículo propone analizar la violencia de género como un problema de salud pública. Numerosos estudios han demostrado que una de cada tres mujeres, en algún momento de su vida, ha sido víctima de violencia sexual, física o psicológica perpetrada por hombres. Durante los últimos decenios, la violencia de género (VG) ha dejado de ser un problema privado y es reconocido y tratado como un problema público; miles de programas se han desarrollado para ayudar a las mujeres, desde albergues y grupos de ayuda legal, hasta grupos de apoyo y servicios de orientación. Activistas y teóricos han comprendido que, aunque esenciales, estos servicios son insuficientes. Para erradicar la violencia de género debemos abordar las causas así como los efectos. Algunos enfoques pueden ser más eficaces que otros; no obstante, la clave para eliminar la VG reside en la participación intersectorial y de la comunidad. Al abordar la VG de manera integral, la posibilidad de prevención se convierte en una realidad y se crean redes sociales para asegurar que las víctimas de la VG reciban la atención y la protección que ellas requieren. Así pues, el objetivo fundamental del trabajo que ahora presentamos es contribuir para que los programas de lucha contra la violencia de género formen parte de los centros de atención primaria en el ámbito de un plan extendido de las políticas de salud pública.

Palabras-clave: Violencia de género; Salud pública; Políticas de género; Desigualdad; Prevención. 


\section{Abstract}

This article aims to analyze gender violence as a public health problem. Numerous studies have demonstrated that one out of three women, at some moment of their life, has been victim of sexual, physical or psychological violence perpetrated by men. During the last decades, gender violence (GV) ceased to be a private problem and is now recognized and treated as a public problem; thousands of programs have been developed to help women, from lodgings, shelters and legal help groups to support groups and guidance services. Activists and theorists have understood that, although essential, these services are insufficient. To eradicate GV we should approach causes as well as effects. Some focuses can be more effective than others; nevertheless, the key to eliminate GV is in intersector and community participation. When approaching GV in an integral way, the possibility of prevention becomes a reality and social networks are created to ensure that GV victims receive the attention and protection they need. Thus, the fundamental objective of this work is to contribute so that the programs that fight against GV become part of primary care centers in the scope of an extended plan of the public health policies.

Keywords: Gender Violence; Public Health; Gender Policies; Inequality; Prevention.

\section{Introducción}

La violencia ejercida contra la mujer es un fenómeno universal que persiste en todos los países del mundo (OMS, 2005). La violencia doméstica, en particular, continúa siendo terriblemente común y es aceptada como "normal" en demasiadas sociedades del mundo (Torres e col., 1996). Así que, erradicar la pandemia de la violencia de género es el verdadero reto del siglo XXI, mucho más que cualquier otro tipo de avance científico, cultural o tecnológico. Si la humanidad aprendiera a detectar, parar y prevenir esta enfermedad histórica, se produciría un punto de inflexión en su evolución: el fatuo "homo sapiens" pasaría a ser persona, ser humano.

El primer paso para afrontar el reto de la erradicación de la violencia de género es aprender a detectarla. La violencia de género suele manifestarse en primer lugar cómo agresión verbal en sus formas más encubiertas. Es muy importante conocer sus mecanismos, porque las secuelas psicológicas de los malos tratos psíquicos son igual o más graves que las de los malos tratos físicos.

Siempre hay alguien que pregunta ¿por qué ella no se va? en lugar de preguntar ¿por qué él la agrede? La sociedad suele poner el centro de gravedad de los malos tratos en la víctima, buscando las razones por las que merece o se deja maltratar, y justifica al agresor diciendo que es un enfermo o un alcohólico.

Las y los profesionales de salud, psicología, asistencia social y otros especialistas involucrados en el tema, tienen un importantísimo papel que jugar en la detección, atención, derivación de la víctima y denuncia si fuera necesario.

La violencia contra la mujer es una medusa de múltiples cabezas y hay que cortarlas todas, una a una, pero al final hay que ir al corazón de la bestia. Ese corazón es el nuestro, porque inevitablemente estamos dentro de una cultura y nos hemos alimentado de su veneno al igual que de su néctar. Para empezar a erradicar la violencia de género podemos empezar a estar atentas a nuestro lenguaje sexista, a la educación que le damos a nuestros hijos, a nuestros roles en la sociedad, a las agresiones verbales solapadas que aguantamos y, sobre todo, a nuestro pensamiento; sí, como cuando sin darnos cuenta nos encontramos sintiendo y actuando como seres humanos de segunda clase. 
Actualmente es muy difícil leer cualquier periódico, oír una emisora de radio o ver algún canal de televisión, donde no se mencionen denuncias por malos tratos.

"[...]Primera víctima de violencia doméstica ${ }^{1}$ en el 2006. Una mujer, cuya edad e identidad no han sido facilitadas, falleció ayer por la tarde en el municipio granadino de Algarinejo tras recibir dos disparos de arma de fuego por la espalda, presuntamente de manos de su pareja [...] La Vanguardia, 2-1-20o6".

La violencia de género, o la violencia dirigida hacia las mujeres, es un fenómeno estructural, es una manifestación producto del machismo que impera en esta sociedad y que está sustentado por la desigualdad y la inequidad, con un dominio de lo masculino sobre lo femenino en lo simbólico y una relación de poder entre los hombres y las mujeres en el mundo cotidiano. Esta desigualdad está relacionada directamente con el Sistema Patriarcal y por ende con la forma en que se conforman las relaciones de poder (Acosta, 2003, 2004). Por lo tanto, la violencia está relacionada con las construcciones sociales que nos sustentan a todos y a todas.

Hablar sobre violencia de género es hablar de cualquier acto, activo o negligente, que vulnere los derechos de las mujeres por el único motivo de "ser mujeres" (Vega, 1999; Evans, 1993). La violencia es ejercida por los hombres en respuesta a la amenaza ficticia que sienten hacia su masculinidad (a su identidad), es una violencia contra todas y cada una de las mujeres de este mundo. Aquí debo establecer una premisa: ningún acto de violencia está justificado, no hay nunca ningún motivo para ejercer la violencia; la violencia siempre es un fracaso. Nuestro fracaso.

Numerosos estudios han demostrado que una de cada tres mujeres, en algún momento de su vida, ha sido víctima de violencia sexual, física o psicológica perpetrada por hombres (Amnistía Internacional, 2001; Bonino Méndez, 1996; Instituto de Investigaciones Feminístas, 2004). Durante los últimos decenios, la violencia de género (VG) ha dejado de ser un problema privado y es reconocido y tratado como un problema público; miles de programas se han desarrollado para ayudar a las mujeres, desde albergues y grupos de ayuda legal, hasta grupos de apoyo y servicios de orientación. Activistas y teóricos han comprendido que, aunque esenciales, estos servicios son insuficientes. Para erradicar la violencia de género debemos abordar las causas así como los efectos. Algunos enfoques pueden ser más eficaces que otros; no obstante, la clave para eliminar la VG reside en la participación intersectorial y de la comunidad (Bosch, 2002). Al abordar la VG de manera integral, la posibilidad de prevención se convierte en una realidad y se crean redes sociales para asegurar que las víctimas de la VG reciben la atención y la protección que ellas requieren.

\section{Situación en el Estado Español}

Si analizamos detalladamente los datos del Instituto Nacional de Estadística Español INE/2007 verificaremos que cada cinco días muere una mujer asesinada por su marido o compañero. Cada año hay más de medio millón de agresiones a mujeres, de todas las edades, dentro del ámbito familiar. Un 18,3\% de mujeres trabajadoras han sufrido alguna vez una agresión de asedio sexual. Un 12,1\% de mujeres, entre 18 y 29 años, y un $11,2 \%$ entre 29 y 39 años, sufren maltrato. De éstas, un $15 \%$ tienen estudios superiores y un $41 \%$ estudios secundarios.

A pesar de los avances obtenidos en la igualdad de derechos, se continúan aplicando desde una perspectiva patriarcal los parámetros de una sociedad estructurada a partir del desequilibrio de las relaciones entre mujeres y hombres (Ferreira, 1992).

En Cataluña y en el resto del Estado español la visualización del problema de la violencia contra las mujeres se ha dado en estos últimos seis años y gracias a la lucha de las asociaciones de mujeres y de los movimientos sociales, se consiguió que pase a ser un problema social lo que hasta ahora era reservado al ámbito doméstico o al ámbito individual de cada mujer.

Desde los acuerdos de los diferentes gobiernos en la IV Conferencia mundial sobre la mujer el año 1995 en Pekín, se comienza este camino hacia el reconocimiento del problema social, y se adopta la definición

1 La violencia doméstica comprende todos aquellos actos violentos (desde el empleo de la fuerza física hasta el acoso o la intimidación) que se producen en el seno de un hogar y que perpetra al menos un miembro de la familia contra otro. Habitualmente, este tipo de violencia no se produce de forma aislada, sino que sigue un patrón constante en el tiempo. Sus principales víctimas son mujeres, niños y personas dependientes (Corsi e col., 2003). 
que todos/as conocemos: "La violencia contra las mujeres es cualquier acto de violencia basado en el género que tiene o puede tener como resultado daños y/o sufrimientos físicos, sexuales y/o psicológicos para las mujeres, y que también tiene en cuenta las amenazas de estos actos, la coacción y la privación arbitraria de la libertad, tanto en la vida pública como en la privada" (Ceballos, 2001).

El Estado español, como sabemos, tiene diferentes identidades, y tanto las asociaciones de mujeres como en general el movimiento feminista y el tejido social están organizados dentro de las diferentes Autonomías: Cataluña, País Vasco, Madrid-Castilla, Andalucía, Galicia, Asturias. Desde 1976 al acabar la dictadura, los movimientos feministas y el asociacionismo se fueron desarrollando en cada lugar, con algunos actos o foros eventualmente de todo el estado. Una de las luchas comunes fue desde el 97 la consigna de una Ley Integral contra la violencia de género, que se consiguió la aprobara el Parlamento español en diciembre del 2004, al ganar los socialistas las elecciones generales en marzo.

\section{Fotograma Socio Antropológico de la Violencia de Género}

Las mujeres que han muerto en manos de sus parejas en los dos últimos años 2006/2005 fueron 62, una cifra considerable, aunque son diez casos menos que los registrados en el año 2004 (año en que entró en vigor la ley integral contra la Violencia de Género), según datos del Instituto de la Mujer (véase Gráfico 1).

\section{Gráfico I - Muertes por Violencia de Género}

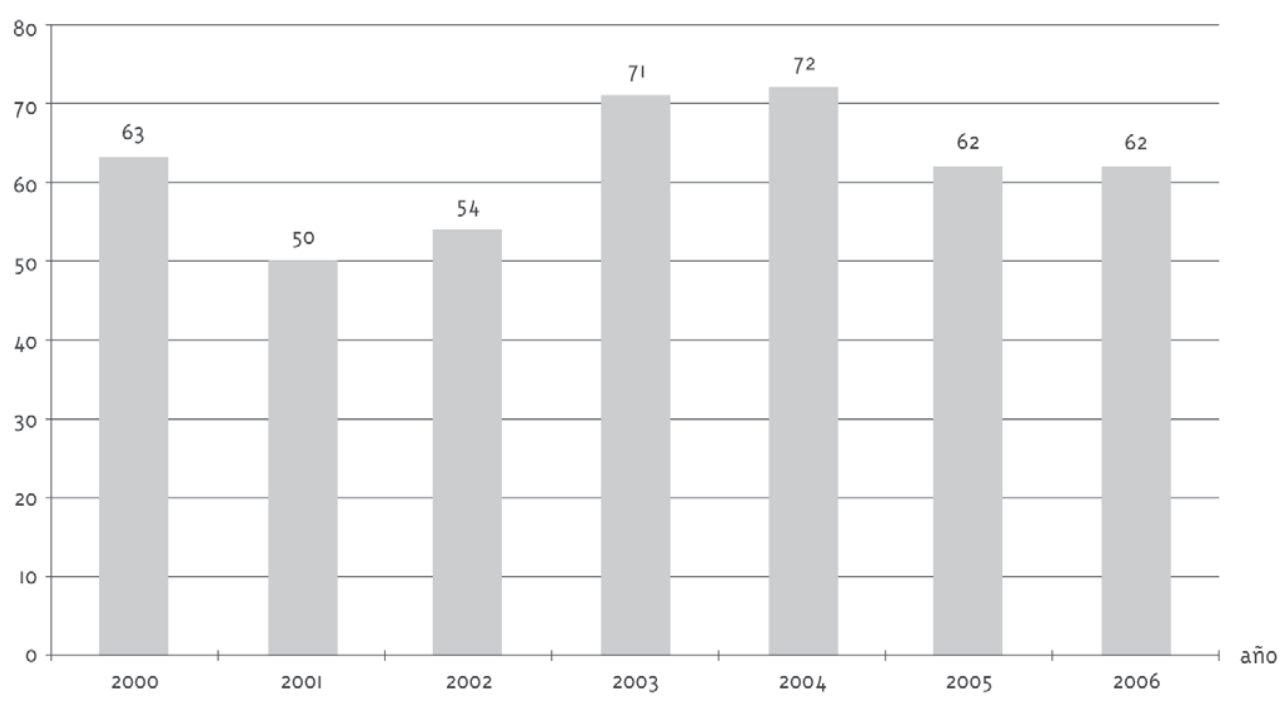

Fuente: Elaboración propia basada en datos del Instituto Nacional de Estadística de España INE/2007 e Instituto de la Mujer.

En el último informe elaborado en 2007 por el Centro Reina Sofía de Valencia² puede leerse: "Los meses más trágicos en cuanto a violencia doméstica se refiere han sido enero, marzo y agosto, con nueve mujeres muertas en cada caso; seguidos de julio, con ocho, y octubre, con seis.
En cuanto a las edades, veintisiete de las víctimas tenían entre 31 y 40 años; trece entre 21 y 30; otras siete entre 41 y 50; nueve eran mayores de 64; cuatro tenían entre 51 y 64 , y dos entre 18 y 20 años (véase Gráfico 2). 


\section{Gráfico 2 - Violencia de género por grupos etários}

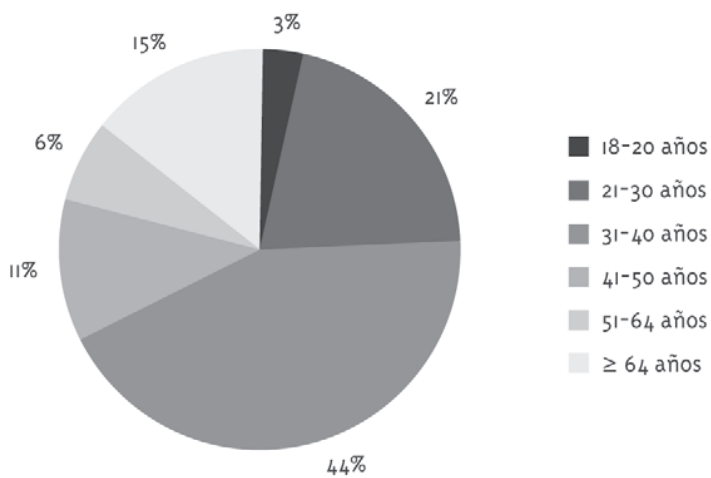

Fuente: Elaboración propia basada en datos del Instituto Nacional de Estadística de España INE/2007 e Instituto de la Mujer.

Respecto a la relación con la pareja, 30 murieron supuestamente en manos de su cónyuge, 12 de su compañero sentimental, 8 de su ex compañero sentimental, 4 de su ex novio, 4 de su ex cónyuge y otras 4 de su novio.

Por nacionalidades, 42 de las mujeres eran españolas, 6 eran ciudadanas de la Unión Europea, 8 eran iberoamericanas, una era ciudadana del este de Europa y 5 eran africanas (véase Gráfico 3).

\section{Gráfico 3 - Violencia de género por nacionalidades}

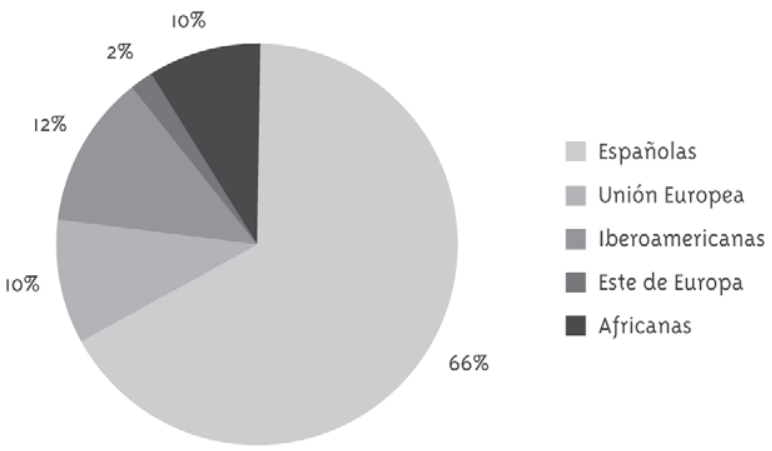

Fuente: Elaboración propia basada en datos del Instituto Nacional de Estadística de España INE/2007 e Instituto de la Mujer.

Por comunidades, Andalucía, con 18 víctimas, es la autonomía con mayor número de muertes, seguida de Cataluña, con 9; la Comunidad Valenciana, con 7; la Comunidad de Madrid, con 5 casos; Castilla-La Mancha, con 4; Castilla y León, País Vasco, Murcia, Canarias y Baleares, con 3 en cada una de ellas; Asturias, con 2, y Aragón y Extremadura, cada una con una mujer fallecida por violencia sexista (véase Gráfico 4).

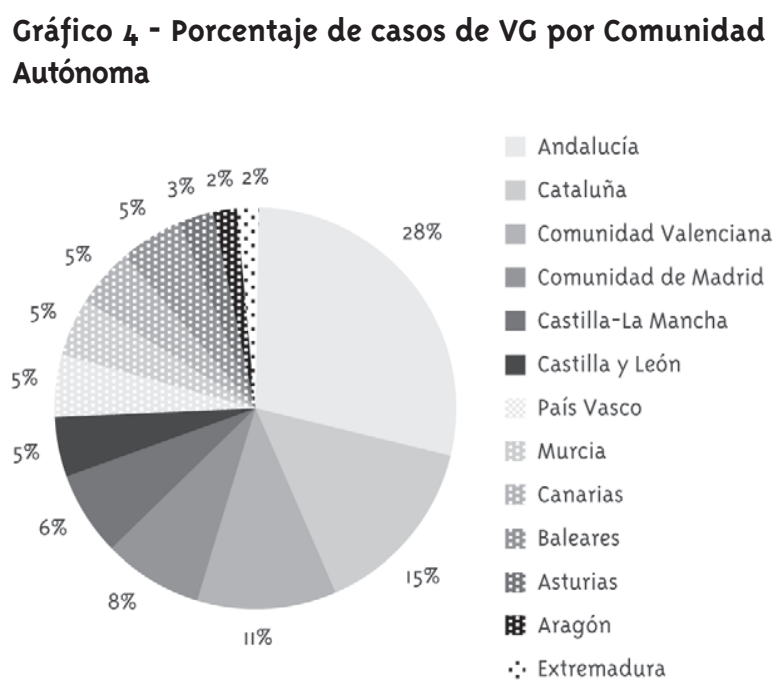

Fuente: Elaboración propia basada en datos del Instituto Nacional de Estadística de España INE/2007 e Instituto de la Mujer.

El informe señala que el 71,5 por ciento de los agresores eran españoles y el resto procedía, principalmente, de países iberoamericanos. Además, menos del 30 por ciento tenía un empleo cualificado y, tras cometer el crimen, un 70 por ciento fue detenido, un 12,5 por ciento se suicidó y otro 15 por ciento lo intentó. Sólo el 2,5 por ciento consiguió huir.

Las cifras alarmantes del informe revelan que los métodos más usuales de asesinato son el uso de armas (un 60 por ciento), el estrangulamiento (un 12,5 por ciento), los golpes físicos (un 7,5 por ciento) y, en el puesto más inusual, con un 2,5 por ciento de incidencia, el degollamiento. Casi en un 8 por ciento de los casos de muertes por violencia doméstica, los hijos estuvieron presentes.

Otro dato curioso que manifiesta el informe es que el mayor número de casos tuvo lugar en ámbitos urbanos donde se produjeron 2,11 víctimas por millón de habitantes frente a las zonas rurales, donde la cifra de asesinatos no superó las 1,84 víctimas por cada millón de ciudadanos.

Aunque la diferencia es poca, se nota que el ritmo de vida de las ciudades, acelerado, influye en que la violencia aflore y se haga notoria. 


\section{Situación Política Respecto a la Ley Integral}

La violencia de género no es un problema que afecte al ámbito privado. Al contrario, se manifiesta como el símbolo más brutal de la desigualdad existente en nuestra sociedad. Se trata de una violencia que se dirige sobre las mujeres por el hecho mismo de serlo, por ser consideradas, por sus agresores, carentes de los derechos mínimos de libertad, respeto y capacidad de decisión. Así define el preámbulo de la ley aprobada por unanimidad el 22 de diciembre de 2004 en el Congreso de Diputados. Esta Ley no es un final de proyecto sino una continuación del proceso iniciado en la última década de presión social. A partir de la lucha de las organizaciones de mujeres se ha creado un renovado movimiento social por los derechos de las mujeres y contra la violencia de género.

La Ley contra la violencia de género se convierte en la primera que aprueba el Congreso de los Diputados. Es una ley pionera en Europa y da un paso muy importante al reconocer y legislar desde la perspectiva del Estado un problema social hasta ahora escondido y avalado en prácticas e interpretaciones de las leyes que daban impunidad a los que ejecutan esta violencia.

\section{Puntos a Resaltar de la ley Integral Contra la Violencia de Género}

\section{Medidas de sensibilización}

La Ley establece medidas de sensibilización e intervención en el ámbito educativo. Se refuerza el ámbito de la publicidad, una imagen que respete la igualdad y la dignidad de las mujeres. La ley insiste en que el sistema educativo ha de tener como un objetivo importante la formación en el respeto a la igualdad entre hombres y mujeres. La Administración revisará los materiales y libros de texto para garantizarlo. En este campo la Ley debería ser más concreta y establecer como obligatorias, en todos los niveles educativos, medidas y métodos para asegurar este principio de igualdad y no discriminación.

Es fundamental la formación específica en las enseñanzas universitarias y técnicas de aquellas profesiones que vayan a intervenir con las mujeres víctimas de violencia, en el ámbito educativo, sanita- rio, psicológico, social y jurídico. Esta formación no es obligatoria por lo que el seguimiento en este punto será de vital importancia para la aplicación de la ley, ya que por experiencia sabemos que desde jueces a médicos o abogados, en muchos casos el diagnóstico es equivocado por falta de formación en el tema.

En las medidas sobre publicidad y medios de comunicación, se considerará ilícita la asociación del cuerpo femenino con un producto concreto. Las asociaciones e instituciones que trabajan a favor de la igualdad podrán solicitar la retirada de un anuncio si lo consideran oportuno.

\section{Medidas de protección y recuperación}

Se apoya a las víctimas por medio del reconocimiento de los derechos como el de información, la asistencia jurídica gratuita y otras de protección social y apoyo económico.

Para garantizar estos derechos, se deberán crear centros de información y asesoramiento, centros de emergencia y casas de acogida, centros de apoyo y recuperación (Quemada e Prieto, 2004). Las Comunidades Autónomas y los Ayuntamientos participarán en la implantación de estos servicios, con asistencia jurídica especializada y gratuita, ayuda económica para recuperarse, formación para el empleo y derechos laborales específicos como adaptación de horarios, movilidad geográfica o rescindir el contrato con subsidio de desempleo. Se crearán Juzgados especializados en cada provincia y capital, en los que se tramitarán tanto asuntos civiles como penales para una misma unidad familiar.

\section{Cuestiones a Mejorar}

El aspecto integral, de actuación global está aún minimizado en la ley aprobada, ya que da más énfasis al aspecto penal y de protección inmediata, que son importantes, que a la Prevención y las medidas sociales, de educación y socialización, que son decisivas. Asimismo desde este aspecto queda más en el marco de la violencia doméstica. Asimismo, desde este aspecto la ley da más énfasis al marco de la violencia doméstica. Elementos a mejorar y para concretar en la discusión en las respectivas leyes de las Comunidades Autónomas:

- Hay que destacar que el reconocimiento de todo tipo de ayuda no debe estar vinculado a la interposición de 
denuncia por parte de la víctima, como hace la ley al exigir la acreditación de esta condición a través de la orden de protección o del informe del Ministerio Fiscal. En muchas ocasiones la mujer no se atreve a denunciar o necesita ayuda antes de la denuncia y por tanto ha de poder acceder a ella.

- Reforzar la detección precoz. No hay que esperar que en la mujer esté muy avanzado el ciclo de la violencia, hay que detectarla antes y poner recursos para evitar que se desarrolle (Marcos, 1998). En el aspecto sanitario hay protocolos de actuación, en los que también faltan recursos, pero en otros campos faltan incluso los protocolos o los circuitos por los que una mujer puede recuperarse antes de llegar a un punto de poner la denuncia por malos tratos.

- Prevenir la victimización de la mujer. La sociedad tiende a culpabilizar a la mujer que quiere salir del pozo, hay que prever instrumentos como la formación de los profesionales y la sensibilización y educación para que la responsabilidad recaiga sobre el maltratador y no sobre la maltratada (Mullender, 200o).

\section{Movilización para Recursos: seguimiento de la Ley}

Ahora lo que perseguimos es su coherencia en la normativización y recursos en su aplicación. Esta ley no es un final de proyecto, sino que se ha de continuar aplicándola y dotándola, a nivel Autonómico y en los Ayuntamientos, para que sea una ley eficiente.

Tenemos una ley, y es muy importante que ha salido con presupuesto, pero hemos de hacer un seguimiento exhaustivo para que se desarrolle y para que se dote del presupuesto adecuado.

Consideramos que es muy decisivo que existan estos presupuestos para que los recursos lleguen a todas las mujeres que padecen maltrato y se pueda prevenir para que no se reproduzca la violencia.

\section{La Violencia de Género en el Ámbito de la Salud Pública}

La violencia de género se ha convertido en un serio problema de salud pública de graves consecuencias para la salud de las mujeres, tanto en la esfera física (lesiones traumáticas, en ocasiones con resultado de muerte, infecciones de transmisión sexual, problemas ginecológicos, embarazos no deseados, lesiones en el feto o recién nacido y bajo peso al nacer, dolor pélvico crónico, hipertensión, cefaleas, síndrome de intestino irritable o fibromialgia) como en la mental (incremento de síntomas y cuadros clínicos de ansiedad, insomnio, depresión clínica y trastorno de estrés postraumático).

El informe mundial de la OMS de 2005 dedicado a la salud de la mujer y violencia doméstica, confirma que entre el $10 \%$ y el $69 \%$ de las mujeres entrevistadas refirieron haber sufrido agresiones físicas por parte de su compañero íntimo masculino en algún momento de sus vidas. Pero, para muchas de estas mujeres, esta agresión física no fue un caso aislado sino que forma parte de un patrón de comportamiento agresivo. Las investigaciones indican que la violencia física en las relaciones íntimas van acompañadas con frecuencia de violencia psicológica, y entre un tercio y la mitad de los casos además se acompañan de abusos sexuales (Vega, 1999; Fuster, 2002).

Este estudio de la OMS titulado Women's Health and Domestic Violence Against Women [Salud femenina y violencia doméstica contra las mujeres], demuestra que las mujeres están más expuestas a la violencia en el hogar que en la calle, lo que tiene graves repercusiones para la salud femenina, también pone de relieve la importancia de sacar a la luz en todo el mundo la violencia doméstica y de tratarla como un grave problema de salud pública. Lamentablemente el abordaje de la violencia de género desde la salud pública y los servicios asistenciales de salud tiene en la actualidad claras limitaciones tanto en la investigación, como en los sistemas de información, la identificación y el tratamiento de las mujeres maltratadas.

Una de las nuevas realidades está en la sensibilización social de cada unos de los actores sociales y en las múltiplas campañas socio-políticas en la cual se inscribe La Campaña Mundial de Prevención de la Violencia de la OMS que ayuda a los gobiernos a definir programas integrales de prevención para atajar todas las formas de violencia, incluida la doméstica. Para la mayoría de los responsables políticos, el mayor problema es que el maltrato siga envuelto en un manto de silencio. Por ello, en el informe se recomiendan un conjunto de intervenciones indispensables para 
modificar los comportamientos y acabar con las desigualdades y normas sociales que perpetúan los malos tratos. También se recomienda integrar planes de prevención de la violencia en las iniciativas que ya estén en marcha dedicadas a la infancia y la juventud, el VIH/SIDA o la salud sexual y reproductiva. Sería necesario formar a los dispensadores de servicios de salud para que sepan reconocer a las víctimas de la violencia doméstica y obrar en consecuencia. Los servicios de atención prenatal, planificación familiar o asistencia post aborto son posibles cauces para atender y apoyar a esas mujeres y derivarlas a otros servicios. Hay que hacer de la escuela un lugar seguro, reforzar los sistemas de apoyo a las víctimas y poner en marcha programas de prevención. La sensibilización de la opinión pública en torno a este problema es un elemento crucial.

\section{Camino por Recorrer para una Detección y Prevención Eficaz de la Violencia de Género}

La violencia de género es todavía un área de investigación muy reciente. La mayoría de los estudios se refieren a la violencia global y se centran en la violencia física, que es más fácil de cuantificar que la violencia de otra naturaleza. Sin embargo, hay poca información sobre la frecuencia y las características de otras formas de maltrato, como el sexual o el psicológico (Lamberti-Sanchez, 2003; Acosta e Acosta, 1999). En España se han publicado pocos estudios epidemiológicos sobre el tema (Casado, 1995; Ceballos, 2001; Quemada e Prieto, 2004)).

Existen iniciativas como la del Departamento de Salud Pública de la Universidad de Alicante de la Red temática de Investigación de Salud y Género que ha realizado un estudio epidemiológico descriptivo que explora la distribución temporal de la mortalidad por violencia del compañero íntimo (VCI) en España e identifica posibles agrupamientos temporales en la ocurrencia de muertes por VCI. Para llevarlo a cabo, se han basado en el registro de la Federación de Mujeres Separadas y Divorciadas (1998-2003), cuyos datos proceden de noticias de prensa publicadas sobre el tema.

Para este estudio se seleccionaron a las mujeres muertas por violencia por parte del compañero íntimo y se excluyeron los casos por otro tipo de relacio- nes o por desconocidos. La detección de casos resulta un punto crítico. No obstante, la evidencia científica resulta insuficiente para recomendar el uso de determinados instrumentos dada la baja sensibilidad y especificidad de los que se han desarrollado, así como a los problemas derivados de la elevada proporción de falsos negativos y positivos que pueden ocasionar calificaciones inapropiadas y actitudes punitivas.

Por otra parte, la recogida de datos se realiza todavía de manera descoordinada y con poco rigor por parte de los diferentes agentes que participan en la asistencia a las personas maltratadas (hospitales, juzgados, comisarías). La creación de estos registros es reciente y no están unificados.

En cuanto a la asistencia sanitaria, la falta de formación y desarrollo de habilidades en la entrevista clínica por parte de los profesionales sanitarios dificulta el descubrimiento de nuevos casos de violencia doméstica. Ello se debe a que muy pocos médicos, enfermeras y demás personal de salud cuentan con la sensibilidad y la formación necesarias para reconocer la violencia como la causa subyacente de los problemas de salud de las mujeres. La inexistencia de un espacio físico idóneo que proporcione seguridad y confianza a las víctimas y el escaso tiempo del que disponen los sanitarios para cada paciente, son otros factores que pueden complicar el diagnóstico. Evidentemente, el sector salud no puede llevar a cabo estos cambios por sí solo, pero debería hacer realidad este potencial y desempeñar una función destacada en la prevención de la violencia.

\section{¿Qué se Puede Hacer desde la Asistencia Sanitaria por las Víctimas de la Violencia de Género?}

El rol de los profesionales de la salud en la prevención, detección precoz y asistencia a las víctimas de la violencia de género puede ser muy importante, ya que está comprobado que las mujeres que sufren malos tratos utilizan los servicios sanitarios con más frecuencia (González e Rosaura, 2001). Así, la inclusión de preguntas específicas sobre abusos en la historia clínica rutinaria (de la misma manera que se pregunta repetida y regularmente sobre el hábito de fumar, el consumo de alcohol, el peso o el ejercicio físico) puede resultar un método efectivo para la detección de 
casos de violencia doméstica (Ravazzola, 1997). Por otra parte, también sería necesario fomentar la declaración de los casos de maltrato en los servicios de urgencias.

Además de contribuir a poner de manifiesto el problema a partir de la recogida de datos, los sanitarios pueden aportar nuevos métodos de identificación del maltrato, promover la formación de profesionales y elaborar guías de recursos especializadas.

Finalmente, ante el conocimiento del abuso, deben responder lo más eficazmente posible a las necesidades de las mujeres afectadas, informándolas sobre la existencia de recursos específicos que respondan a sus necesidades. Resulta muy relevante para las víctimas una simple información sobre la existencia de servicios especializados y cómo contactarse con ellos. A través de estas recomendaciones, el sanitario puede ayudar a reducir el sentimiento de aislamiento y estigmatización que sufren estas mujeres.

Dada la morbilidad y mortalidad asociada con la violencia doméstica, el abordaje desde la salud pública debería contemplar como puntos importantes la integración de métodos de identificación dentro de la rutina en la atención sanitaria, además del trabajo interdisciplinar de diferentes actores, fomentar la creación de registros específicos, tanto de morbilidad como de mortalidad, la elaboración de protocolos y la formación de los profesionales sanitarios.

\section{Conclusiones}

La violencia es una manifestación compleja de un sistema de violencias intersectorial izadas (física, psicológica, moral, económica, sexual, laboral, esclavitud, institucional, sanitaria, educacional) e interrelacionadas entre sí (Cárdenas, 1999). La Violencia Estructural es aquella legitimada y que legitima al mismo tiempo el sistema social y económico en que vivimos. La violencia estructural se sustenta en la creación de constructos de desigualdad y de inequidad, que se radicalizan en la construcción de los fundamentalismos (económicos, sociales, religiosos, de clase, de raza, etc.). Esta desigualdad y este desequilibrio se observan en el acceso a los recursos y a los servicios de salud, que aunque en determinados contextos (estado español) se considera universal, no es en ningún modo real.
Los datos de mujeres fallecidas víctimas por la violencia machista reflejan cifras que se salen del ámbito doméstico para afectar a toda la sociedad. La violencia contra las mujeres es un abuso contra los derechos humanos. Por ello es responsabilidad del Estado prestar asistencia, protección y garantizar justicia a las víctimas de la violencia doméstica. Precisamente, la nueva ley abarca tanto los aspectos preventivos, educativos, sociales y judiciales, como los asistenciales y de atención posterior a las víctimas. Además, se establecen por primera vez medidas de sensibilización e intervención en el ámbito sanitario para mejorar la detección precoz y la atención de las víctimas.

\section{Referencias}

ACOSTA, M. Agresión a la mujer: realidades y mitos. Barcelona: Ed. Barcelona, 2003.

ACOSTA, M. El rompecabezas: anatomia de un maltratador. Barcelona: Ares y Mares, 2004.

ACOSTA, M.; ACOSTA, J. A. Agresión a la mujer: maltrato, violación y acoso. Granada: Comares, 1999.

AMNISTÍA INTERNACIONAL. Cuerpos rotos, mentes destrozadas: tortura y malos tratos a mujeres. Madrid, 2001.

BONINO MÉNDEZ, L. Micromachismos: la violencia invisible en la pareja. Madrid: Generalidad Valenciana, Dirección General de la Mujer. 1996.

BOSCH, E. La voz de las invisibles: las víctimas de un mal amor que mata. Madrid: Cátedra, 2002.

CÁRDENAS, E. J. Violencia en la pareja. Buenos Aires: Grani, 1999 .

CASADO, C. Malos tratos conyugales a mujeres en el área de Sevilla. Sevilla: Instituto Andaluz de la Mujer, 1995.

CEBALLOS, E. La violencia doméstica: análisis sociológico, dogmático y de derecho comparado. Granada: Comares, 2001.

CORSI, J. et al. Maltrato y abuso en el ámbito doméstico: fundamentos teóricos para el estudio de la violencia en las relaciones familiares. Buenos Aires: Paidós, 2003. 
EVANS, P. Verbal abuse: survivors speak out on releationship and recovery. Holbrook, Mass: Adams Media Corporation, 1993.

FERREIRA, G. B. Hombres violentos, mujeres maltratadas. Buenos Aires: Sudamericana, 1992.

FUSTER, E. Las víctimas invisibles de la violencia familiar: el extraño iceberg de la violencia doméstica. Madrid: Paidós, 2002.

GONZÁLEZ, M.; ROSAURA, S. H. J. Violencia en las parejas jóvenes: análisis y prevención. Madrid: Pirámide, 2001.

INSTITUTO DE INVESTIGACIONES FEMINÍSTAS. Violencia de género y sociedad: una cuestión de poder. Madrid: Ayuntamiento de Madrid, 2004.

LAMBERTI-SANCHEZ, V. (Org.). Violencia familiary abuso sexual. Buenos Aires: Ed. Universidad de Argentina, 2003.
MARCOS, L. Las semillas de la violencia. Madrid: Espasa, 1998.

MULLENDER, A. La violencia doméstica: una nueva visión de un viejo problema. Barcelona: Paidós, 2000. OMS - ORGANIZACIÓN MONDIAL DE LA SALUD. Estudio multipaís de la OMS sobre salud de la mujer y violencia doméstica contra la mujer. Suiza, 2005.

QUEMADA, C.; PRIETO, P. La violencia contra las mujeres: prevención y detección. Madrid: Díaz de Santos, 2004

RAVAZZOLA, M. C. Historias infames: los maltratados en las relaciones. Buenos Aires: Paidós, 1997.

TORRES, P.; ESPADA, F. J. La violencia en casa. Madrid: Aguilar, 1996.

VEGA, R. Las agresiones familiares en la violencia doméstica. Pamplona: Aranzádi, 1999. 